\section{Voltammetrie, zyklische und inverse}

\section{T. Arndt}

Bioscientia Institut für Medizinische Diagnostik GmbH, Ingelheim, Deutschland

Synonym(e) Gleichstrom-Voltammetrie; Dreiecksspannungs-Voltammetrie

Englischer Begriff voltammetry; cyclic voltammetry; stripping voltammetry; triangular wave voltammetry

Definition Bezeichnung für ein elektrochemisches Analysenverfahren, bei dem Strom-Spannungs-Kurven (Voltammogramme) zur qualitativen und quantitativen Analyse erzeugt werden.

Beschreibung Bei der Voltammetrie (nicht zu verwechseln mit der $>$ Voltametrie, einem elektrochemischen Verfahren zur Titrations-Endpunkterkennung) benutzt man eine in die Probenlösung eintauchende, polarisierte Elektrode und misst die bei Spannungsänderung gegen eine unpolarisierte Bezugselektrode auftretenden Ströme. Die resultierenden StromSpannungs-Kurven sind durch eine Stromspitze bei einem bestimmten Potenzial geprägt. Diese Spitzenpotenziale sind analytspezifisch und in Tabellenwerken gesammelt. Der Spitzenstrom ist wiederum ein quantitatives $\mathrm{Ma}$ für die Konzentration des sog. Polarisators in der Analysenlösung.

Bei der zyklischen Voltammetrie wird das Potenzial innerhalb weniger Sekunden linear verändert und nach Erreichen des Maximalwertes linear zurück auf den Ausgangspunkt gebracht. Trägt man das Potenzial gegen die Zeit auf, ergibt sich eine Dreiecksform, weshalb die Methode auch als Dreieckspannungs-Voltammetrie (nach der IUPAC-Nomenklatur ,[cyclic] triangular wave voltammetry“) bezeichnet wird. Diese Variante der Voltammetrie wird zur Untersuchung der Reversibilität von Reaktionen eingesetzt.

Die Voltammetrie unterscheidet sich von der verwandten - Polarographie dadurch, dass die Indikatorelektrode ein hängender Quecksilbertropfen, also stationär ist, während bei der Polarographie der Quecksilbertropfen wirklich tropft (und dadurch die Elektrodenoberfläche regelmäßig erneuert wird).

$\mathrm{Zu}$ Einzelheiten und den vielfältigen Variationen der Voltammetrie s. Lehrbücher der Analytischen Chemie.

Die Voltammetrie hat im klinisch-chemischen Routinelabor keine Bedeutung. Sie erlaubt jedoch, wie viele elektrochemische Analyseverfahren, äußerst sensitive und spezifische Analysen von Ionen. Ihr Hauptanwendungsgebiet ist deshalb die Spurenanalytik, z. B. in der Wasser- und Umweltchemie. Sie kann hier als ein von der zumeist eingesetzten Atomabsorptions- und Atomemissionspektrometrie unabhängiges Analyseverfahren für Gegenanalysen (analog zu den Bestätigungsanalysen in der Drogenanalytik) herangezogen werden.

\section{Literatur}

Falbe J, Regitz M (Hrsg) (1992) Römpp Chemie Lexikon. Georg Thieme Verlag, Stuttgart/New York

Latscha HP, Linti GW, Klein HA (2004) Analytische Chemie. Chemie Basiswissen III. Springer, Berlin/Heidelberg/New York 